\title{
Modeling and Simulation of Atomic Clock Clean-up System
}

\author{
Chao $\mathrm{Xu}^{1,2}$, Bo Li ${ }^{1,2}$, Junliang Liu ${ }^{1}$ and Huabing $\mathrm{Wu}^{1}$ \\ ${ }^{1}$ National Time Service Center, Chinese Academy of Sciences, Xi' an, China \\ ${ }^{2}$ University of the Chinese Academy of Sciences, Beijing, China
}

\begin{abstract}
The atomic clock clean-up system aims at improving the short term frequency stability while maintaining the good long term frequency stability of the atomic clock itself. A simulation model for the atomic clock clean-up system is established, which is paramount importance for design, simulation, and selection of the suitable equipments for atomic clock clean-up system. Based on the phase locked loop(PLL) structure, the phase noise of oscillator, phase detector noise and voltage controlled oscillator(VCO) quantization noise which influence the performance of the system are modeled respectively in this simulation model. Then, a clean-up experiment has been designed for a widely used cesium atomic clock to test the performance of the atomic clock clean-up system. The experiment results show that the short term frequency stability of the cesium atomic clock under test is improved with the reasonable selection of the system parameters, and its short term frequency stability which is not inferior to the hydrogen maser. This simulation model can also be applied to the simulation of other types of PLL systems by changing the corresponding noise model.
\end{abstract}

Keywords-atomic clock; clean-up; pll

\section{INTRODUCTION}

Since National Physical Laboratory (NPL) developed the first practical atomic clock in $1955^{[1]}$, it has been widely used in many fields, such as wireless communication, satellite navigation, aerospace, astronomical observation and timefrequency measurement. Atomic clock has gradually become an essential component for these fields which demand high precision frequency and time reference.

With the rapidly development of science and technology, the demand of excellent short-term frequency stability is rising. For example, the Time Keeping System(TKS) of GPS Block IIR satellite ${ }^{[2]}$ uses a phase locked loop (PLL) to take advantages of the good long-term frequency stability of a rubidium atomic clock and the excellent short -term frequency stability of a voltage controlled oscillator(VCO). According to the evaluation results of GPS Master Control Station, the estimated range deviation (ERD) which is an estimate of the range error the users would see using the broadcast ephemeris and clock has been reduced obviously since TKS started running ${ }^{[3]}$.

At present, there are some clean-up equipment designed by relevant manufacturers, such as $4145 \mathrm{C}$ produced by Microsemi ${ }^{[4]}$, VCH-317 produced by VREMYA-CH ${ }^{[5]}$, A6ANF produced by Quartzlook ${ }^{[6]}$, and Clean-up Oscillator produced by Timetech ${ }^{[7]}$, which have been used in many applications. However, there are few researches on the modeling, simulation and performance analysis of the atomic clock clean-up system. In this paper, a simulation model is established, which is based on PLL structure, to verify and analyze the performance of the atomic clock clean-up system.

\section{CleAn-UP Modeling AND SimUlation}

PLL technology is the foundation of atomic clock clean-up system. The atomic clock will be "cleaned" by this high performance PLL, and the short term frequency stability performance of output signal will be improved.

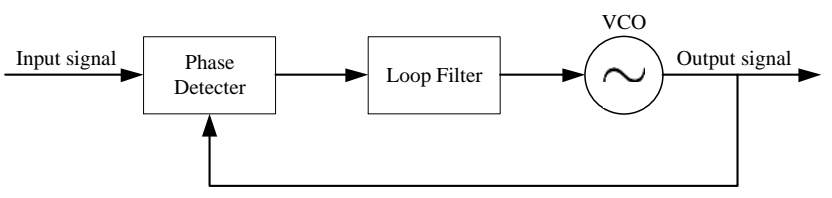

FIGURE I.

BLOCK DIAGRAM OF A PLL

Block diagram of a typical PLL is shown in Figure I. It contains 3 essential units: phase detector, loop filter and voltage controlled oscillator. The phase detector compares the phase of the periodic input signal with the VCO signal, and outputs the phase difference between the two signals. Then, the phase difference is filtered by the loop filter, and the output of the loop filter is used as the control voltage of VCO to reduce the phase error between the input signal and the $\mathrm{VCO}^{[8]}$.

The clean-up performance of atomic clock is mainly related to the phase noise of the atomic clock, the phase noise of VCO, the noise of phase detector, and the quantization noise of VCO. On the basis of the block diagram in Figure I. , the simulation model of atomic clock clean-up system can be established as shown in Figure II.

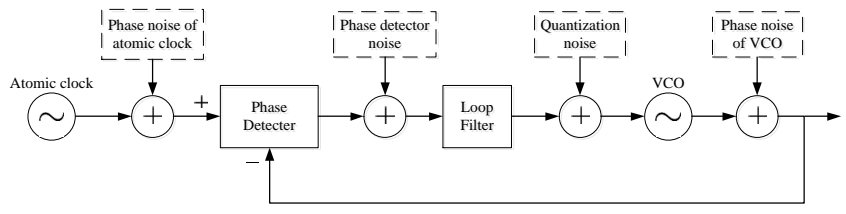

FIGURE II. SIMULATION MODEL OF ATOMIC CLOCK CLEAN-UP SYSTEM 


\section{MOdeling OF Phase NoISE OF OSCILlator}

\section{A. The Phase Noise of Oscillator}

The phase noise of the atomic clock and VCO can be simulated by the same model. The oscillator's phase noise can be well approximated as superposition of independent noises with different power-law types of spectral densities. Its power spectral density function is expressed as ${ }^{[9]}$

$S_{x}(f)=\frac{1}{4 \pi^{2}}\left(h_{-2} f^{-4}+h_{-1} f^{-3}+h_{0} f^{-2}+h_{1} f^{-1}+h_{2}\right)(1)$

$h_{\alpha}$ is a constant, $\alpha=-2,-1,0,1,2$ are corresponding to 5 different types noise respectively. There are random walk frequency noise, flicker frequency noise, white frequency noise, flicker phase noise and white phase noise. This function is shown graphically in Figure III.

It can be observed the different slopes of the different types of noise in the timing error spectral distribution. The random walk frequency is a low frequency noise which has a great influence on the long term frequency stability, while the white phase is the floor noise that is mandatory in the short term.

\section{B. Clock Error Modeling}

The characterization of phase noise of oscillator can be reflected by clock error. Clock error is defined as the clock timing error caused by the instantaneous phase change of the oscillator:

$$
x(t)=\int_{0}^{t} y\left(t^{\prime}\right) d t^{\prime}
$$

The normalized frequency error $y(t)$ is given by

$$
y(t)=\frac{f(t)-f_{o}}{f_{o}}
$$

where $f_{o}$ is the nominal frequency. A widely used definition for the measure of frequency stability is known as Allan variance, its expression is

$$
\begin{aligned}
\sigma_{y}^{2}(\tau) & =\frac{1}{2(N-1)} \sum_{k=1}^{N-1}\left(\bar{y}_{k+1}-\bar{y}_{k}\right)^{2} \\
& =\frac{1}{2(N-1)} \sum_{k=1}^{N-2}\left(\frac{x(k+2)-2 x(k+1)+x(k)}{\tau}\right)^{2}(4)
\end{aligned}
$$

where $\tau$ is the averaging period, $N$ is sample number. Allan variance can be translated from the power spectral density of the timing error. The types of clock error with their contributions to spectral density $S_{x}(f)$ and Allan variance are shown in TABLE I. The high-frequency cut-off $f_{h}$ is defined as the upper limit in the spectral bandwidth of the system.

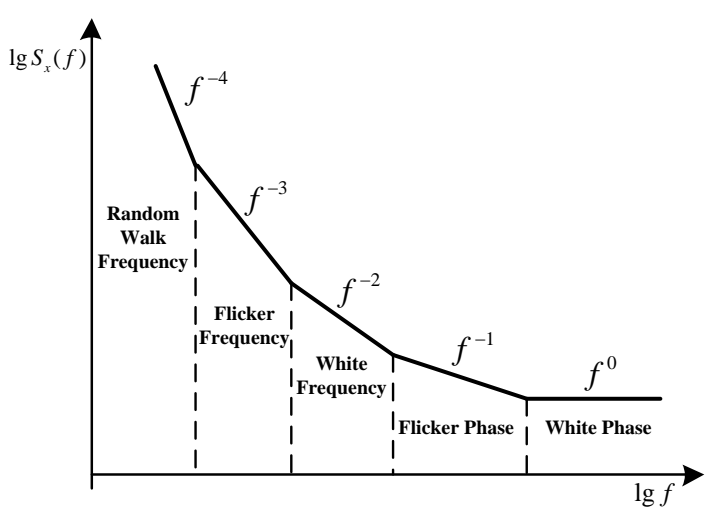

FIGURE III

POWER SPECTRAL DENSITY OF TYPICAL CLOCK NOISE

In general, flicker phase noise is not considered because it can be neglected in the analysis of clock error ${ }^{[10]}$. Set the noise parameter of other 4 types phase noise as

$$
\begin{aligned}
& A_{w p}=\sqrt{\frac{3 h_{2} f_{h}}{4 \pi^{2}}}, A_{w f}=\sqrt{\frac{h_{0}}{2}} \\
& A_{f f}=\sqrt{2 \ln 2 h_{-1}}, A_{r w}=\sqrt{\frac{4 h_{-2} \pi^{2}}{6}}
\end{aligned}
$$

TABLE I. TYPES OF CLOCK NOISE WITH THEIR CONREBUTIONS TO SPECTTRAL DENSITY AND ALLAN VARIANCE

\begin{tabular}{|c|c|c|}
\hline Type of noise & $S_{x}(f)$ & $\sigma_{y}^{2}(\tau)$ \\
\hline White Phase & $h_{2}$ & $\frac{3 h_{2} f_{h}}{4 \pi^{2} \tau^{2}}$ \\
\hline Flicker Phase & $h_{1} f^{-1}$ & $h_{1} \frac{6+3 \ln \left(2 \pi f_{h} \tau\right)-\ln 2}{4 \pi^{2} \tau^{2}}$ \\
\hline White Frequency & $h_{0} f^{-2}$ & $\frac{h_{0}}{2 \tau}$ \\
\hline Flicker Frequency & $h_{-1} f^{-3}$ & $2 h_{-1} \ln (2)$ \\
\hline Random Walk Frequency & $h_{-2} f^{-4}$ & $\frac{2 h_{-2} \pi^{2} \tau}{3}$ \\
\hline
\end{tabular}

Then the frequency stability of the oscillator can be specified as a function of Allan variance for each type of noise, as it is shown in TABLE II.

TABLE II. DEFINITION OF THE FERQUENCY STABILITY

\begin{tabular}{|c|c|}
\hline Type of noise & Allan Variance \\
\hline White Phase & $\sigma_{y_{-} w p}^{2}(\tau)=\left(A_{w p} \cdot \tau^{-1}\right)^{2}$ \\
\hline White Frequency & $\sigma_{y_{-} w f}^{2}(\tau)=\left(A_{w f} \cdot \tau^{-1 / 2}\right)^{2}$ \\
\hline Flicker Frequency & $\sigma_{y_{-} f f}^{2}(\tau)=A_{f f}^{2}$ \\
\hline Random Walk Frequency & $\sigma_{y_{-} r w}^{2}(\tau)=\left(A_{r w} \cdot \tau^{1 / 2}\right)^{2}$ \\
\hline
\end{tabular}

\section{Simulation of Clock Error}

The random sequences which obey uniform distribution would be a good choice for simulating the nominal frequency $y(t)$ of each type of noise, it can be generated using these equations ${ }^{[1]]}$ : 


$$
\begin{gathered}
y_{\mathrm{wp}, \mathrm{i}}=A_{\mathrm{wp}} \tau_{s}^{-1} k_{\mathrm{wp}}\left(\operatorname{rand}_{i}-\operatorname{rand}_{i-1}\right) \\
y_{\mathrm{wf}, \mathrm{i}}=A_{\mathrm{wf}} \tau_{s}^{-1 / 2} k_{\mathrm{wp}}\left(\operatorname{rand}_{i}\right) \\
y_{\mathrm{rw}, \mathrm{i}}=y_{\mathrm{rw}, \mathrm{i}-1}+A_{\mathrm{rw}} \tau_{s}^{1 / 2} k_{\mathrm{rw}}\left(\text { rand }_{i}\right)
\end{gathered}
$$

where 'rand' is a uniform variable in the interval $[-1,1], N$ is the number of simulated points at a sampling frequency of $1 / \tau_{s}$. The generation of these three types of errors are convenient and without large computational load. However, the flicker frequency, which is an important component of clock error simulation, may be complex in terms of large computational load. According to the method described in [12], the flicker frequency can be generated by using the following algorithm:

$y_{\mathrm{ff}, \mathrm{i}}=A_{\mathrm{ff}} k_{\mathrm{ff}}\left[i^{-2 / 3} \operatorname{rand}_{1}+(i-1)^{-2 / 3} \operatorname{rand}(2)+\cdots+\operatorname{rand}_{i}\right](9)$

This method works well and provides very good results, but it requires managing all the previous samples in each step.

In order to remain the Allan variance of the generated random sequences unchanged, normalization constants $k$ must be introduced when generating these four types of nominal frequency error. The constants for each type of noise are provided in TABLE III. [11]

The proposed noise simulation method using (6)-(9), allows the generation of real nominal frequency error. However, in some applications, it is necessary to fit a given error behavior while keeping the statistical characterization of the clock noise. Therefore, a method for fitting noise parameters using overdetermined nonlinear equations is adopted by taking the Allan deviation of the given clock as input ${ }^{[13]}$. Where Allan deviation is the square root of Allan variance.

$\left\{\begin{aligned} \sigma_{y}\left(\tau_{1}\right) & =\sqrt{\left(A_{\mathrm{wp}} \cdot \tau_{1}^{-1}\right)^{2}+\left(A_{\mathrm{wf}} \cdot \tau_{1}^{-1 / 2}\right)^{2}+A_{\mathrm{ff}}^{2}+\left(A_{\mathrm{rw}} \cdot \tau_{1}^{1 / 2}\right)^{2}} \\ \sigma_{y}\left(\tau_{2}\right) & =\sqrt{\left(A_{\mathrm{wp}} \cdot \tau_{2}^{-1}\right)^{2}+\left(A_{\mathrm{wf}} \cdot \tau_{2}^{-1 / 2}\right)^{2}+A_{\mathrm{ff}}^{2}+\left(A_{\mathrm{rw}} \cdot \tau_{2}^{1 / 2}\right)^{2}} \\ \vdots & \\ \sigma_{y}\left(\tau_{m}\right) & =\sqrt{\left(A_{\mathrm{wp}} \cdot \tau_{m}^{-1}\right)^{2}+\left(A_{\mathrm{wf}} \cdot \tau_{m}^{-1 / 2}\right)^{2}+A_{\mathrm{ff}}^{2}+\left(A_{\mathrm{rw}} \cdot \tau_{m}^{1 / 2}\right)^{2}}\end{aligned}\right.$

where $\sigma_{y}(\tau)$ is the Allan deviation of different $\tau, m \geq 4$. Newton iterative method is one of the most effective methods for solving nonlinear equations. The optimal noise parameters $A=\left[A_{w p}, A_{w f}, A_{f f}, A_{r w}\right]$ can be obtained by solved by these overdetermined nonlinear equations.

Once the noise parameters are calculated, the timing error, i.e., the clock error as a function of the time, is obtained as

$$
x_{i}=x_{i-1}+\left(y_{i}^{W P}+y_{i}^{W F}+y_{i}^{F F}+y_{i}^{R W}\right) \tau_{s}
$$

TABLE III. NORMALISATION CONSTANT

\begin{tabular}{|c|c|}
\hline Type of noise & Constant Value \\
\hline White Phase & $k_{w p}=1$ \\
\hline White Frequency & $k_{w f}=\sqrt{3}$ \\
\hline Flicker Frequency & $k_{f f}=2.26$ \\
\hline Random Walk Frequency & $k_{w p}=\sqrt{6}$ \\
\hline
\end{tabular}

A simulation experiment has been performed to test the behavior of the proposed algorithm. TABLE IV. and Figure IV. show the results between the Allan deviation of a cesium atomic clock HP5071A which taken as input and Allan deviation of simulated clock error.

\section{TABLE IV. ALLAN DEVIATION OF A HP5071A CESIUM ATOMIC} CLOCK

\begin{tabular}{|c|c|c|}
\hline \multirow{2}{*}{$\tau / \mathrm{s}$} & \multicolumn{2}{|c|}{$\sigma_{y}(\tau)$} \\
\cline { 2 - 3 } & original & simulated \\
\hline 10 & $3.00 \times 10^{-12}$ & $2.53 \times 10^{-12}$ \\
\hline 20 & $2.03 \times 10^{-12}$ & $1.77 \times 10^{-12}$ \\
\hline 40 & $1.38 \times 10^{-12}$ & $1.25 \times 10^{-12}$ \\
\hline 100 & $8.7 \times 10^{-13}$ & $7.88 \times 10^{-13}$ \\
\hline 200 & $5.99 \times 10^{-13}$ & $5.59 \times 10^{-13}$ \\
\hline 400 & $4.06 \times 10^{-13}$ & $3.94 \times 10^{-13}$ \\
\hline 1000 & $2.7 \times 10^{-13}$ & $2.51 \times 10^{-13}$ \\
\hline 2000 & $1.93 \times 10^{-13}$ & $1.80 \times 10^{-13}$ \\
\hline 4000 & $1.12 \times 10^{-13}$ & $1.32 \times 10^{-13}$ \\
\hline 10000 & $5.2 \times 10^{-14}$ & $1.0 \times 10^{-13}$ \\
\hline
\end{tabular}

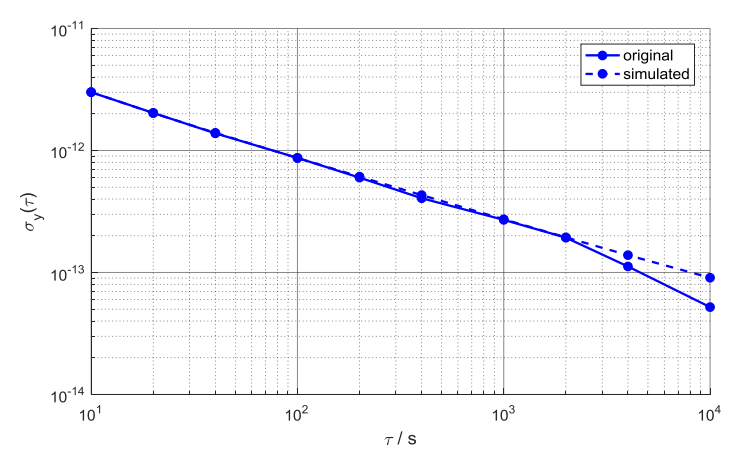

FIGURE IV.

\section{ALLAN DEVIATION OF SIMULATION EXPERIMENT}

The above experiment results indicate that the Allan deviation of the clock error data generated by the proposed method is very close to the input Allan deviation of HP5071A.This method allows the deep analysis of the noise behavior of the high precision oscillator without losing the actual random behavior, and solves one of the main problems in the simulation of atomic clock clean-up system.

\section{Modeling of Noise of Phase Detector And VCO}

For a well-designed PLL system, the phase noise should mainly originate from the oscillator. However, the noise of 
phase detector and VCO may also influence its actual performance.

\section{A. Modeling of Phase Detector Noise}

The characteristics of phase detector noise are different with different types of phase detectors. For example, the TKS of Block IIR satellite uses a time interval counter which has a resolution of $1.67 \mathrm{~ns}$ because of its $600 \mathrm{MHz}$ driving frequency ${ }^{[14]}$. The statistical characteristics of the output of this phase detector obey the uniform distribution. Therefore, a random sequences within the range of $[-1.67,1.67] \mathrm{ns}$ can be used to generate phase detector noise of TKS.

For the atomic clock clean-up system, low phase measuring accuracy will introduce excessive system noise to the system, and resulting in the degradation of clean-up performance. As a result, the dual mixer time difference(DMTD) system with a picoseconds phase measurement accuracy can be used as the phase detector of the clean-up system to guarantee the performance of the clean-up system. The DMTD equipment produced by Symmetricom company is widely used in many time keeping laboratories. Its floor noise experiment shows that the floor noise obey the Gauss distribution with the standard deviation of $10^{-13}$ level. The experiment results are shown in Figure V.

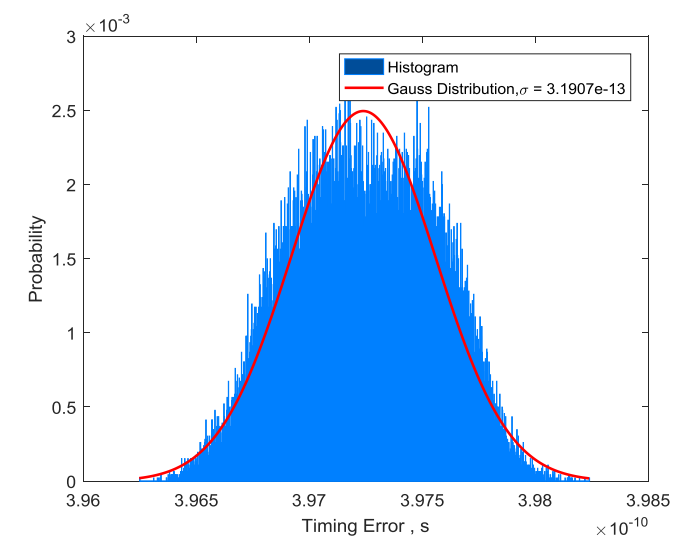

FIGURE V.

STATISTICS OF SYMMETRICOM DMTD EQUIPMENT

According to the above statistics, Gauss white noise random sequences would be used as the model of phase detector noise in the atomic clock clean-up system.

\section{B. Modeling of VCO Quantization Noise}

The commonly used model of quantization noise is additive white noise with equal probability density to the linear system. For digital phase locked loop (DPLL), a high precision DAC module is used to generate the control voltage to adjust the VCO frequency. Therefore, the resolution of the DAC module will determine the magnitude of the quantization noise.

If OSA 8607 option E oscillator is used as the VCO of the atomic clock clean-up system, for example, its typical normalized frequency adjustment range is $\left[-10^{-7}, 10^{-7}\right]$, and input control voltage range is $[0,10] \mathrm{V}$, the output frequency will be equal to its nominal value when the input control voltage is $5 \mathrm{~V}^{[15]}$. Suppose that the DAC module is $\mathrm{N}$ bits, then the normalized frequency adjustment resolution of OSA 8607 option $\mathrm{E}$ will be $10^{-7} / 2^{N-1}$. Therefore, a random sequences which obey uniform distribution would be used as the model of VCO quantization noise.

\section{SimUlation RESUlTS}

An experiment was carried out to test the proposed simulation model. The Allan deviation results of each component are shown in Figure VI.

This experiment adopts the Allan deviation of the cesium atomic clock HP5071A which introduced in chapter III as the input to generate clock error of reference atomic clock. An OSA 8607 option E oscillator is used as the VCO, whose Allan deviation can be obtained from its technical manual ${ }^{[15]}$. Setting the standard deviation of Gauss white noise which simulates the phase detector noise as $3 \times 10^{-13}$, and setting the normalized frequency adjustment resolution as $10^{-7} / 2^{19}$.

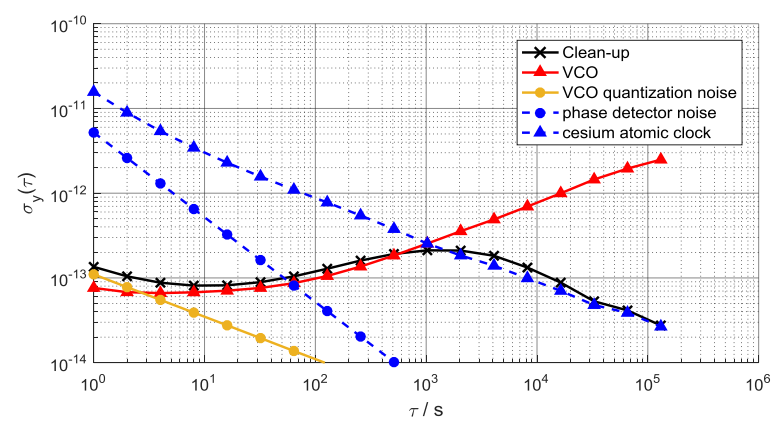

FIGURE VI. ALLAN DEVIATION RESULTS OF EACH SIMULATION SIGNAL

From the simulation results, it can be observed that the output signal of the clean-up system not only maintains the long term characteristics of the cesium atomic clock, but also obtains excellent short term characteristics of VCO. The atomic clock clean-up system improves the short term frequency stability of the cesium atomic clock used for the experiment, and its short term frequency stability is not inferior to the hydrogen maser.

\section{CONCLUSIONS}

A simulation model of an atomic clock clean-up system based on PLL is proposed. The oscillator noise, phase detector noise and VCO quantization noise are modeled as the major noise of this simulation model respectively. This simulation model proposed in this paper solves one of the main problems in the design and analyze the atomic clock clean-up system. The influence of different noise on clean-up result can also be analyzed through this simulation model. This simulation model is based on PLL, so it is also applicable to most of the digital PLL simulation.

\section{REFERENCES}

[1] Pultarova, T, "Changing times [atomic clocks]," Engineering \& Technology 10.6(2015):76-77. 
[2] Rawicz, H. C., M. A. Epstein, and J. A. Rajan. "The time keeping system for GPS block IIR." Annual Precise Time \& Time Interval Applications \& Planning (1993).

[3] Petzinger, Mr John, M. R. Reith, and M. T. Dass. "Enhancements to the GPS block IIR timekeeping system." Enhancements to the Gps Block Iir Timekeeping System (2002).

[4] 4145C Ultra-Clean Phase-Locked Oscillator. California: Symmetricom, 2003.

[5] Real-Time Atomic Clock Combiner VCH-317 Operational Manual. Novgorod: Vremya-ch.

[6] Active Noise Filter Atomic Clock Clean up Oscillator A6-ANF. England: Quartzlock.

[7] Clean-up Oscillator. Stuttgart: TimeTech GmbH,2006.

[8] Gardner, Floyd M. Phaselock techniques. John Wiley \& Sons, 2005.

[9] Barnes, James A., et al. "Characterization of Frequency Stability." IEEE Transactions on Instrumentation and Measurement IM-20.2(2010):105120.

[10] Harting, A. "Considering clock errors in numerical simulations." IEEE Transactions on Instrumentation \& Measurement 45.3(1996):715-720.

[11] Diez, J., P. D'Angelo, and A. Fernández. "Clock Errors Simulation and Characterisation." Proceedings of International Technical Meeting of the Satellite Division of the Institute of Navigation (2006):815-821.

[12] Barnes, J. A., and D. W. Allan. "A statistical model of flicker noise."Proceedings of the IEEE 54.2(1966):176-178.

[13] Chao Xu, Bo Li, Junliang Liu, Huabing Wu, Yonghui Hu. " A modified method for clock error simulation of high precision frequency source". Journal of Astronautics 38.9(2017):998-1004.

[14] Wu, Dr Andy. "Evaluation of GPS Block IIR Time Keeping System for Integrity Monitoring." Performance Evaluation of the Gps Block Iir Time Keeping System (2007).

[15] Over Controlled Crystal Oscillator 8607. Neuchatel: Oscilloquartz, 2002. 